\title{
Radiological Images on Personal Computers: Introduction and Fundamental Principles of Digital Images
}

\author{
Thurman Gillespy III and Alan H. Rowberg
}

\begin{abstract}
This series of articles will explore the issues related to displaying, manipulating, and analyzing radiological images on personal computers (PC). This first article discusses the digital image data file, standard PC graphic file formats, and various methods for importing radiological images into the $P C$.

Copyright 1993 by W.B. Saunders Company
\end{abstract}

KEY WORDS: personal computer, digital image, network, image file format, TIFF.

$\mathbf{T}$ HERE ARE THREE noteworthy trends in today's radiology department: (1) high performance personal computers are becoming ubiquitous, (2) there are increasing numbers of digital images being generated, and (3) high performance computer networks are poised to connect our personal computers and the imaging modalities that generate these digital images.

As a consequence of the convergence of these three trends, there is increasing interest in using personal computers (PCs) to display, analyze and manipulate radiological images. Displaying and working with radiological images on PCs are technically difficult because PCs were not designed for these functions. This review series will discuss the technical and user interface issues that arise when PCs are used for this purpose.

This article will review basic principles of digital images, common graphic file formats found on PCs, and methods of importing radiological images into the PC. Future articles will discuss how 16-bit radiological images can be displayed on PCs with 8-bit graphic hardware, image processing and analysis, image compression and storage, and a review of the many potential uses of the $\mathrm{PC}$ in working with radiological images. The emphasis of the series will be on what can be accomplished by the radiologist or imaging scientist with equipment that is likely to appear on an office desktop (as opposed to dedicated, high performance workstations). In the next article, an application pro- gram that operates on Macintosh computers (Apple Computer, Cupertino, CA) will be presented and made available that illustrates many of the principles discussed in the series.

In addition, we believe encouraging the readers to consider the problems of displaying radiological images on their personal computers will help them become more aware of the issues and problems related to radiology workstation design, image transfer, and image storage. We also believe the continued technical advancement of high performance PCs will reduce the need for expensive, special purpose hardware to display radiological images. Finally, there are many articles in this journal and other journals that present new methods of image processing, analysis, display, and compression. Many of these methods are developed on computer systems that are not available to most readers. We hope the availability of an open, low-cost radiology image display application that operates on the widely available Macintosh computer would encourage these authors to implement their software in a manner that allows it to be used with this application.

\section{TERMINOLOGY}

Personal computer (PC): A computer system that is likely to appear on a radiologist's or secretary's desktop, commonly used for word processing, simple database management, and graphics.

IBM compatible personal computer (IBM-PC) (International Business Machines Corp, New York, NY): A computer system either compatible with or manufactured by IBM, typically

From the Department of Radiology, University of Washington, Seattle, $W A$.

Address reprint requests to Thuman Gillespy $I I I, M D$, Department of Radiology, SB-05, University of Washington, 1956 NE Pacific St, Seattle, WA 98195.

Copynght 1993 by W.B. Saunders Company

0897-1889/93/0602-0004\$03.00/0 
using either the DOS, OS/2, or the Windows operating environments.

High performance PC: A system with at least the performance of a 80386 or 68020 central processing unit (CPU), capable of rapidly displaying 256 gray shades.

Macintosh computer: System manufactured by (or compatible with) Apple Computer Company. Although many different Macintosh systems have been produced, in this series we will be exclusively referring to color Macintoshes (Macintosh II, or better) with at least 8-bit color graphic capabilities.

\section{THE DIGITAL IMAGE DATA FORMAT}

Although radiologists use the phrase "digital images" as if it was a single entity, digital images are actually a group of entirely different objects that are loosely connected under this term. Usually, the object is not an image unless it can be viewed, and it is probably in an analog form at the time of viewing. The brightness of a cathode ray tube display (computer screen), for instance, may be an analog of the digital representation of an image. Typically, a higher digital value is associated with a brighter spot in the corresponding place in the image. When an image is printed on film with a laser printer, or photographed onto film using a conventional multiformat camera, the density of grains of silver on the film are also an analog of the digital representation of the image.

Commonly, what we mean when we speak of a digital image is a computer representation of that image, essentially a string of bytes that are stored in the computer in a data structure. Usually, this is a disk file on the computer's magnetic hard disk. However, it may also be in memory, on a magnetic tape, or on an optical disk. Although this block of information is actually a series of bytes, we commonly speak of it as having picture elements, called pixels, which are represented as one or two bytes ( 8 or 16 bits) of information. The image data itself is commonly in a raster form, with each separate row of the image being stored successively in the data structure. Although these data might be thought of as being stored in a matrix form with rows and columns of pixels, this storage format is usually not used in the computer. However, it is convenient for users and programmers to continue to think about the image as if it were stored in a matrix form.

The minimum size of the image data file in bytes is calculated by the following equation:

$$
\text { image data }=\mathrm{n}^{*} \mathrm{~m}^{*} \mathrm{k}
$$

where $n$ is the number of rows, $m$ is the number of columns (also referred to as the $x$ and $y$ dimensions of the image), and $k$ is the number of bytes used to store each pixel (either 1 or 2 ). The size of the image data files for various digital imaging modalities is shown in Table 1.

Patient demographic and scanning parameter information may be associated with the image as an image header. Although this might be a separate data structure or file, it is commonly stored at the beginning of the data file, taking up a fixed amount of space. This is convenient when transferring files because the image data always goes along with the corresponding image header. Some images also contain information after the image data, and this portion of the image file is termed the image footer. To extract the examination and patient information, the data format of the header and (if present) the footer must be known. However the image can still be viewed, even if this format is not known, by skipping the header and only loading the image data for display. The final image file size (in bytes) can be calculated:

$$
\begin{aligned}
\text { image file size }= & \text { [header }] \\
& + \text { image data }+ \text { [footer }]
\end{aligned}
$$

where [] denotes optional items.

\section{Image Dynamic Range and Bit Depth}

The difference between the largest and smallest image data value is the dynamic range or contrast resolution of the image. The dynamic range is closely related to the bit depth of the image. The bit depth is the largest possible dynamic range of an image expressed as a power of 2. For most digital imaging modalities the bit depth ranges from 8 to 13 (Table 1). The relationship between the dynamic range and the bit depth of an image is shown in the following equation:

$$
0<\text { dynamic range } \leq 2^{\text {n }}-1
$$


Table 1. Image File Parameters for Common Image Types

\begin{tabular}{|c|c|c|c|c|}
\hline Image Type & Image Matrix* & File Size $†$ & Bit Depth & $\begin{array}{l}\text { Images per } \\
\text { 80M HD }\end{array}$ \\
\hline \multirow[t]{2}{*}{ Nuclear medicine } & $64 \times 64 \times 1$ & 4,096 & 8 & 19,500 \\
\hline & $128 \times 128 \times 1$ & 16,384 & 8 & 4,800 \\
\hline MRI & $256 \times 256 \times 2$ & 131,072 & $13 \xi$ & 600 \\
\hline CT & $512 \times 512 \times 2$ & 524,288 & 12 & 150 \\
\hline \multicolumn{5}{|l|}{ Digitized radiograph } \\
\hline $1 \mathrm{~K}$ & $1,024 \times 842 \times 2$ & $1,724,416$ & $\left.12\right|_{j}$ & 45 \\
\hline $2 \mathrm{~K}$ & $2,048 \times 1,684 \times 2$ & $6,897,664$ & & 11 \\
\hline Computed radiograph & $2,140 \times 1,760 \times 2$ & $7,532,800$ & 10 & 10 \\
\hline
\end{tabular}

* Rows $\times$ columns $\times$ number of bytes per pixel. The matrix dimensions are typical, but will vary for digitized radiographs and computed radiographs. In the $C$ programming language, 1 byte pixels are cast as unsigned char, and 2 byte pixels are cast as unsigned short integers.

IIn bytes, without any associated header or footer files.

ҒApproximate number of uncompressed images that could be stored on an 80 -megabyte hard disc.

§The actual dynamic range for MRIs can vary tremendously depending on the signal intensity at the receiver coil and the type of puising sequence. We have seen rapidly acquired gradient echo images with dynamic ranges of $\sim 100$, and surface coil images with dynamic ranges of $>6,000$.

|Because of noise, the usable dynamic range of digitized radiographs and computed radiographs is typically 1 or 2 bits less than the bit depth of the image data.

Abbreviations: MRI, magnetic resonance image; CT, computed tomorgraph.

where $n$ represents the bit depth of an image. For example, ultrasound and nuclear medicine images are commonly stored as 8-bit (1-byte) integers per pixel. Thus, at most 256 image values $\left(2^{8}=256\right)$ per pixel can be stored. Because radiological images are typically stored as unsigned integers (ie, negative numbers are not used), the image values can range from 0 to 255 . On the other hand, computed tomography (CT) images have a bit depth of 12 . Thus, at most 4,096 different CT image values $\left(2^{12}=4,096\right)$ per pixel can be stored. Because the Hounsfield scale sets the density of water to zero, the range of possible CT image values extends from $-1,024$ to 3,071 Hounsfield units (HU). However, because unsigned integers are used to store the CT image data, the CT image values as stored on disc or tape must be converted to $\mathrm{HU}$ as shown by the following equation:

$$
\mathrm{HU}=\mathrm{CT} \text { image data value }-1,024
$$

The distinction between the bit depth and the pixel data size of an image is occasionally confused in the imaging community. The confusion is illustrated by the following question: Are CT images 12-bit or 16-bit images? The correct answer depends on your frame of reference. If you are interested in the bit depth or maximum possible dynamic range of the image data, they are 12-bit images. However, if you are interested in the size of each pixel as stored in the computer memory or on disc or tape, they are 16-bit (two byte per pixel) images. A proper understanding of both concepts is essential when developing programs to display and analyze radiological images.

\section{6-Bit Pixel Byte Order}

The order of the two bytes that comprise 16-bit pixels (and of the 4 bytes that comprise 32-bit values) varies among different computer systems (Table 2). On Macintosh and other computers, the high-order byte precedes (has a lower memory address) than the low-order byte. This arrangement is known as a "big endian" byte order (Fig 1). Conversely, IBM-PCs, VAX computers, and others have a "little endian" byte order in which the low-order byte precedes the high-order byte (Fig 2). If the byte order of the image acquisition system is different than the byte order of the PC (or other image display system) of interest, then the byte order must be swapped before the image can be displayed, analyzed, or manipulated. 
Table 2. Big and Little Endian Computer Systems and Image Acquisition Systems

\begin{tabular}{lll}
\hline \multicolumn{1}{c}{ System } & \multicolumn{1}{c}{ Big Endian } & \multicolumn{1}{c}{ Little Endian } \\
\hline CPUs & $\begin{array}{c}\text { Motorola 680 } \\
\text { series }\end{array}$ & $\begin{array}{c}\text { Intel } 808 \times, \\
80 \times 86 \text { series }\end{array}$ \\
$\begin{array}{l}\text { Personal computers, } \\
\text { workstations }\end{array}$ & $\begin{array}{c}\text { Macintosh, Sun, } \\
\text { NeXT, Amiga }\end{array}$ & IBM-PC \\
$\begin{array}{l}\text { Other computer } \\
\text { systems }\end{array}$ & Data General & VAX, PDP-11 \\
$\begin{array}{l}\text { CT scanners } \\
\text { MRI scanners }\end{array}$ & GE 9800 & Siemens \\
X-ray digitizers & & Siemens \\
Computed radiography & Fuji & Dupont \\
\hline
\end{tabular}

Abbreviations: CPUs, central processing units; CT, computed tomography; MRI, magnetic resonance image.

\section{COMMON GRAPHIC AND IMAGE FILE FORMATS}

Images are commonly stored in standard data file formats, and there are a wide variety of computer programs available for manipulating and displaying images in many of these standard formats. There are also programs available to change a file from one image format to another while preserving its image content and perhaps some of the associated image header. Common graphic file formats on Macintosh computers include PICT and MacPaint (Table 3). Common graphic file formats on IBM compatible PCs include PCX. File formats widely supported on both systems include tagged interchange file format (TIFF), CompuServe graphic

High Memory

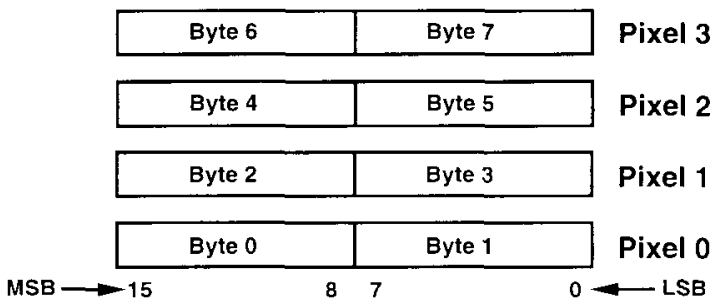

Low Memory

Fig 1. Big endian systems. Bytes 0, 2, 4, and 6 are the high-order bytes, and bytes $1,3,5$, and 7 are the low-order bytes. $L S B$, least significant bit, $M S B$, most significant bit.
High Memory

\begin{tabular}{|c|c|c|}
\hline Byte 6 & Byte 7 & Pixel 3 \\
\hline Byte 4 & Byte 5 & Pixel 2 \\
\hline Byte 2 & Byte 3 & Pixel 1 \\
\hline Byte 0 & Byte 1 & Pixel 0 \\
\hline 7 & & \\
\hline
\end{tabular}

Fig 2. Little endian systems. Bytes 1, 3, 5, and 7 are the high-order bytes and bytes $0,2,4$, and 6 are the low-order bytes. $\angle S B$, least significant bit, $M S B$, most significant bit.

interchange format (GIF), and encapsulated postscript (EPS).

The use of a standard image format is convenient for the user because the same display program may be used to display any image, regardless of the original source of the image. For instance, if the user sets up a system which converts both CT images and magnetic resonance images (MRIs) to the TIFF format, then any computer program that supports TIFF may be used to display these images. The specifics of the CT and MRI header and image formats need be dealt with only in the process of acquiring these images. In all subsequent steps, these images may be simply treated as TIFF images and processed without having to handle specific details related to the source of the images.

\section{TIFF File Format}

The TIFF file format is a popular standard for storing and describing raster image data. TIFF is capable of describing bilevel, gray scale, palette-color and full-color image data in several color spaces. The standard is portable and does not favor any particular operating system, file system, compilers, or processor. TIFF includes several different compression schemes and is designed to be extensible. The baseline TIFF standard version 6.0 only supports 4 and 8-bit gray scale images. Although 16-bit images are possible within the extended version of the standard, we have not encountered any examples of 16-bit radiological images stored as TIFF files. A complete description of the TIFF stan- 
Tabie 3. Common Personal Computer Graphic File Formats

\begin{tabular}{|c|c|c|c|c|}
\hline File Format & System & Comment & Gray Scale & 16-bit \\
\hline MacPaint & Macintosh & Bit mapped, bilevel & No & No \\
\hline PICT, PICT-2 & Macintosh & $\begin{array}{l}\text { QuickDraw based; supported by many Mac } \\
\text { programs }\end{array}$ & Yes & No \\
\hline $\begin{array}{l}\text { SuperPaint, Pixel-Paint, } \\
\text { PhotoShop, ThunderScan }\end{array}$ & Macintosh & Various formats native to specific programs & Yes & No \\
\hline TIFF & Macintosh, IBM-PC & $\begin{array}{l}\text { System independent; supported by many } \\
\text { graphics and image processing programs }\end{array}$ & Yes & Yes $^{*}$ \\
\hline EPS & Macintosh, IBM-PC & $\begin{array}{l}\text { PostScript based; not commonly used by } \\
\text { image processing or display programs }\end{array}$ & Yes & No \\
\hline GIF & IBM-PC, Macintosh & $\begin{array}{l}\text { System independent standard developed } \\
\text { for CompuServe }\end{array}$ & Yes & No \\
\hline $\mathrm{PCX}$ & IBM-PC & $\begin{array}{l}\text { Supported by PC Paintbrush, Ventura Pub- } \\
\text { lisher, and Aldus PageMaker }\end{array}$ & Yes & No \\
\hline PIC, Dr Halo, & IBM-PC & Various formats native to specific programs & Yes & No \\
\hline Targa, Deluxe Paint II & ATL Medical, HP & Ultrasound images on magneto-optical disk & Yes & No \\
\hline DEFF & ATL, HP & Ultrasound images on magneto-optical disk & Yes & No \\
\hline Interfile & Nuclear medicine & Image data exchange format & Yes & No \\
\hline
\end{tabular}

*Extended TIFF can describe 16-bit gray scale images, but we have seen no examples of such files to date.

Abbreviations: TIFF, tagged interchange file format; PC, personal computer; EPS, encapsulated postscript; GIF, graphic interchange format; DEFF, data exchange file format; ATL, Advanced Technology Laboratories; HP, Hewlett Packard.

dard is available from Aldus Corporation (see Further Readings, below).

A TIFF file begins with an 8-byte image file header (IFH) that points to one or more image file directories (IFDs). The first two bytes of the IFH describe whether the data is stored in little or big endian format. An IFD contains information about the image as well as pointers to the actual image data. The IFD contains a series of 12-byte directory entries that consist of a unique tag identifier followed by information relevant to the tag. For example, tags numbered 256 and 257 describe the rows and columns of the image data matrix, respectively. The required fields to describe a gray scale image are listed in Table 4.

\section{IMPORTING IMAGES INTO THE PC}

In radiology, importing images into the $\mathrm{PC}$ has traditionally been very difficult because the majority of digital image sources do not make provision for delivering images to the user in a form or format that can be used by PCs. Because CT scanners are commonly standalone devices, they are typically designed without a great deal of interface capability, if any. Even teleradiology systems, which are designed to communicate images, commonly use a proprietary data format, so that images cannot be easily moved to other display systems. The common image acquisition methods are summarized in Table 5 .

\section{Video Frame Grabber}

The most flexible image acquisition device is a video frame grabber, which is an image capture system on a computer card which is placed into the user's computer system. It is connected to a video source, such as a television camera, and the software associated with the card is used to capture images and store them in disk files on the hard disk. Because the TV camera can be pointed at any image source, including a TV monitor, an x-ray film view box, a photograph of a medical image in a journal, or any other scene, this system is very flexible. The

Table 4. Required Fields for TIFF Gray Scale Images (Baseline tagged interchange file format 6.0)

\begin{tabular}{lccc}
\hline \multicolumn{1}{c}{ TagName } & Decimal & Hexadecimal & Value \\
\hline ImageWidth & 256 & $0 \times 100$ & \\
ImageLength & 257 & $0 \times 101$ & \\
BitsPerSample & 258 & $0 \times 102$ & 4 or $8^{*}$ \\
Compression & 259 & $0 \times 103$ & 1 or 32,773 \\
Photometric & & & \\
$\quad$ Interpretation & 262 & $0 \times 106$ & 0 or 1 \\
StripOffsets & 273 & $0 \times 111$ & \\
RowsPerStrip & 278 & $0 \times 116$ & \\
StripByteCount & 279 & $0 \times 117$ & \\
XResolution & 282 & $0 \times 11 \mathrm{~A}$ & \\
YResolution & 283 & $0 \times 11 \mathrm{~B}$ & \\
ResolutionUnit & 296 & $0 \times 128$ & 1 or 2 or 3 \\
\hline
\end{tabular}

* The baseline TIFF requirement does not allow for 16 bit grayscale images, but such images are possible with extended TIFF files. 
Table 5. Characteristics of Image Acquisition Methods

\begin{tabular}{|c|c|c|c|c|c|c|}
\hline & $\begin{array}{c}\text { Spatial } \\
\text { Resolution }\end{array}$ & $\begin{array}{l}\text { Contrast } \\
\text { Resolution }\end{array}$ & $\begin{array}{l}\text { Grayscale } \\
\text { Accuracy }\end{array}$ & Artifacts & Convenience & Cost \\
\hline Video frame grabber & Medium to high & High & Fair & Possible & High & Medium \\
\hline Magnetic tape & High & High & High & No & Low & Low \\
\hline Digita: network & High & High & High & No & High & Moderate \\
\hline Laser or CCD film scanner & High & High & Good & Rare & Moderate & High \\
\hline Video camera film digitizer & Low & Fair & Poor & Moderate & Low & Medium \\
\hline
\end{tabular}

Abbreviation: CCD, charge-coupled device.

method is limited by the capability of the camera itself, and image quality may not be adequate for all applications. This device may also be connected to standard video tape players, to acquire images in those situations when the images have been stored on video tape, such as in some real time ultrasound applications.

In some circumstances, it is desirable to connect a video frame grabber directly to a medical imaging device, such as a CT scanner. Because the video characteristics of these scanners are not the same as standard TV, special image capture systems are required. These are more expensive than the ones used with standard video, because the higher resolution capability of the video system used requires greater band width in the electronic circuits. There are also special features required to synchronize the image capture with the individual pixels of the image display on the radiographical image modality.

\section{Film Digitizers}

Laser film digitizers have become popular in recent years because they do not require a TV camera and lens, so there is no need to focus a camera on the image. The high stability and wide dynamic range of the components used may allow them to capture images with up to, or exceeding, 1,000 shades of gray, with good reproducibility and excellent linearity. Because of the nature of the scanning process, the image obtained is accurately rectangular, and does not have the barrel-shaped distortion which is common with camera lenses. In some newer scanners, charge-coupled devices (CCDs) are used to capture the image. These are solid state sensors that are capable of recording a portion of an image and converting it into digital format, for direct input into computer circuits. Typically, the $\mathrm{x}$-ray image is illuminated from the back, and the CCD is scanned across the front and used to record brightness of the transmitted light.

\section{Tape and Disk Archive Systems}

In the early days of CT scanning, very few devices contained digital input/output facilities, and archiving was routinely done on reels of magnetic tape. The most common method of transferring these images to other computers was by writing a program on the remote computer to read the image archive tape. This was practical because these archive tapes were routinely available, and no special arrangements were necessary to initiate the transfer of an image. However, the reel of tape had to be moved to the other computer, and specialized software had to be written to read the image from the tape, extract the image from other associated image on the tape, and write it to a disk file. In the late 1970 s nearly all minicomputers that were used in medical imaging applications had such a tape drive, and they were a commonly used back up storage device. With the advent of PCs in the 1980s, floppy disks and smaller tape cartridges have become much more common. Now, as optical disks are being used as image archive devices on many scanners, they may also be used as an image transfer medium. However, the availability of both the hardware and the software required to read the optical disks is typically a problem because the images are usually in a format which has been highly optimized for the storage of medical images, and they is not are not standardly used in the computer industry.

\section{Ethernet Networks}

There is a growing use of Ethernet computer networks to connect personal computers and image acquisition systems in radiology depart- 
ments. The most common data transfer proto$\mathrm{col}$ used for Ethernet networks is the transmission control protocol/internet protocol (TCP/ IP). If the host computer of the image acquisition system supports TCP/IP, images can be easily transferred to PCs on the same network equipped with widely available telecommunications software. The Radiology Department at the University of Washington currently has an Ethernet network that connects all the department PCs (mostly Macintoshes), workstations and research computers, a GE 9800 HiLite Advantage CT scanner (General Electric, Milwaukee, WI) and two General Electric Signa 1.5T MRI scanners. We can transfer CT and MRI images to our Macintosh computers using telecommunications software that supports either telnet or file transfer protocol (ftp). Telnet and ftp are two file transfer options in TCP/IP networks. Software currently in use include VersaTerm (Synergy Software, Reading, PA), NCSA Telnet (free software, National Center for Supercomputing Applications, Champaign, IL) and Fetch (free software, Dartmouth University). Although this software allows us to transfer images, there is no ability to intelligently query the CT or MRI scanners about what images are currently available. Therefore, some a priori knowledge of the patient and the types of images that were obtained is required before the image transfer process can begin.

The transfer of images over TCP/IP networks will improve when the Digital Imaging and Communications in Medicine (DICOM) 3.0 standard becomes widely supported. This standard has been jointly developed by the American College of Radiology (ACR) and National Electrical Manufacturers Association (NEMA). Earlier versions, which did not support TCP/IP networks, were known as ACR-NEMA versions 1 and 2. The DICOM 3.0 standard simplifies the process of image transfer over TCP/IP networks. The ability to intelligently query image acquisition devices or central image archives is supported. It should also be possible to initiate image transfer from the image acquisition device to a PC. However, it will probably be more difficult to obtain PC telecommunications software that supports the DICOM 3.0 standard than software that supports TCP/IP.

\section{FURTHR READING}

Huang HK: Elements of Digital Radiology. Englewood Cliffs, NJ, Prentice-Hall, 1987. This excellent comprehensive and moderately technical book covers the basics of digital radiology; and image acquisition, compression, processing, and display.

Hunter TB (ed): The Computer in Radiology. Rockville, MD, Aspen Systems Corporation, 1986. Although some of the material is outdated, this book is still an excellent introduction to digital imaging and the basics of digital image processing.

Baxes GA: Digital Image Processing. Denver, CO, Cascade, 1988. A good nontechnical introduction to the basics of digital imaging and image processing.

Newell JD, Kelsey CA: Digital Imaging in Radiology: New York, NY, Churchill Livingstone, 1990. This book discusses clinical applications and technical aspects of digital imaging with a chapter on image compression and reconstruction.

Kuni CC: Introduction to Computers \& Digital Processing in Medical Imaging. Chicago, IL, Year Book Medical, 1988. A general and decidedly nontechnical introductory book on medical imaging.

Lindley CA: Practical Image Processing in C. New York, NY, John Wiley \& Sons, 1991. A programming book that covers image acquisition, manipulation, storage, and compression for MS-DOS computers. TIFF 5.0 (not the current standard) is discussed in an appendix. Very useful source code is available for an additional fee.

Embree PM, Kimble B: C Language Algorithms for Digital Signal Processing. Englewood Cliffs, NJ, Prentice Hall, 1991. A moderately technical programmíng book on signal processing (which includes image processing). Source code disc (MS-DOS) is included with the book.

Aldus Corporation: TIFF, Revision 6.0. Seattle, WA, 1992. A very well written specification manual on TIFF that is mandatory reading for programmers interested in working with TIFF files. There are also good sections on image compression. Available from: Aldus Developers Desk, Aldus Corp, 411 First Ave S, Seattle, WA 98104-2871. Also available in PostScript form on CompuServe (GO ALDSVC, Message Section \#10) and on the Stanford ftp server (sumex-aim.stanford.edu, info-mac/tech/tiff-revision60. hqx). 